\title{
Search for MeV dark photons in a light-shining-through-walls experiment at CERN
}

\author{
S.N. Gninenko \\ Institute for Nuclear Research, Moscow 117312
}

(Dated: July 11, 2018)

\begin{abstract}
In addition to gravity, there might be another very weak interaction between the ordinary and dark matter transmitted by $U^{\prime}(1)$ gauge bosons $A^{\prime}$ (dark photons) mixing with our photons. If such $A^{\prime}$ s exist, they could be searched for in a light-shining-through-a-wall experiment with a highenergy electron beam. The electron energy absorption in a calorimeter (CAL1) is accompanied by the emission of bremsstrahlung $A^{\prime}$ s in the reaction $e Z \rightarrow e Z A^{\prime}$ of electrons scattering on nuclei due to the $\gamma-A^{\prime}$ mixing. A part of the primary beam energy is deposited in the CAL1, while the rest of the energy is transmitted by the $A^{\prime}$ through the "CAL1 wall" and deposited in another downstream calorimeter CAL2 by the $e^{+} e^{-}$pair from the $A^{\prime} \rightarrow e^{+} e^{-}$decay in flight. Thus, the $A^{\prime}$ s could be observed by looking for an excess of events with the two-shower signature generated by a single high-energy electron in the CAL1 and CAL2. A proposal to perform such an experiment to probe the still unexplored area of the mixing strength $10^{-5} \lesssim \epsilon \lesssim 10^{-3}$ and masses $M_{A^{\prime}} \lesssim 100 \mathrm{MeV}$ by using 10-300 GeV electron beams from the CERN SPS is presented. The experiment can provide complementary coverage of the parameter space, which is intended to be probed by other searches. It has also a capability for a sensitive search for $A^{\prime}$ s decaying invisibly to dark-sector particles, such as dark matter, which could cover a significant part of the still allowed parameter space.
\end{abstract}

PACS numbers: 14.80.-j, 12.60.-i, 13.20.Cz, 13.35.Hb

\section{INTRODUCTION}

Understanding of the origin and properties of dark matter is a great challenge for particle physics and cosmology. Several models consider dark sectors of particles that, in addition to gravity, interact with ordinary matter by new very weak forces transmitted by Abelian $U^{\prime}(1)$ gauge bosons $A^{\prime}$ (dark or hidden photons for short), which could mix with our photons. In a class of these models, the $A^{\prime}$ can be massive and the $\gamma-A^{\prime}$ mixing strength may be as large as $\epsilon \simeq 10^{-5}-10^{-3}$, which makes experimental searches for $A^{\prime}$ 's interesting; for a recent review, see Refs. [1, 2] and references therein.

The interaction between $\gamma$ 's and $A^{\prime}$ 's is given by the kinetic mixing [1, 3]

$$
L_{i n t}=-\frac{1}{2} \epsilon F_{\mu \nu} A^{\prime \mu \nu}
$$

where $F^{\mu \nu}, A^{\prime \mu \nu}$ are the ordinary and the dark photon fields, respectively, and parameter $\epsilon$ is their mixing strength. The kinetic mixing of Eq.(1) can be diagonalized resulting in a nondiagonal mass term and $\gamma-A^{\prime}$ mixing. Therefore, any source of photons could produce a kinematically permitted massive $A^{\prime}$ state according to the appropriate mixings. Then, depending on the $A^{\prime}$ mass, photons may oscillate into dark photons-similarly to neutrino oscillations- or, the $A^{\prime}$ 's could decay, e.g., into $e^{+} e^{-}$pairs.

The aim of this work is to show that the still unexplored region of mixing strength $10^{-5} \lesssim \epsilon \lesssim 10^{-3}$ and $A^{\prime}$ masses $M_{A^{\prime}} \lesssim 100 \mathrm{MeV}$ could be probed in a lightshining-through-a-wall-type experiment [1] with a high energy electron beam. If such $A^{\prime}$ s exist, they would be short-lived particles which decay rapidly into $e^{+} e^{-}$pairs with a lifetime $<10^{-10} \mathrm{~s}$. We show that such decays could be observed by looking for events with the exotic signature - two isolated showers produced by a single electron in the detector. Compared to the beam-dump experiment searching for long-lived $A^{\prime}$ s, with the mixing typically $\epsilon \lesssim 10^{-4}$, the advantage of the proposed one is that for the parameter area $10^{-4} \lesssim \epsilon \lesssim 10^{-3}$ and masses $10 \lesssim M_{A^{\prime}} \lesssim 100 \mathrm{MeV}$ its sensitivity is roughly proportional to the mixing squared $\epsilon^{2}$ associated with the $A^{\prime}$ production in the primary reaction and its subsequent fast decay at small distances $\lesssim$ a few $\mathrm{m}$ from the production vertex. While in the former case, it is proportional to $\epsilon^{4}$, one $\epsilon^{2}$ came from the $A^{\prime}$ production, and another $\epsilon^{2}$ is from the probability of $A^{\prime}$ decays in a detector located at a large distance from the dump.

The rest of the paper is organized in the following way. The experimental setup, method of search, background sources, and the expected sensitivity for the decay $A^{\prime} \rightarrow e^{+} e^{-}$are discussed in Sec. II. The search for the $A^{\prime} \rightarrow$ invisible decay mode, background and the expected sensitivity are discussed in Sec. III. Section IV contains concluding remarks.

\section{THE EXPERIMENT TO SEARCH FOR $A^{\prime} \rightarrow e^{+} e^{-}$DECAYS}

The process of the dark photon production and subsequent decay is a rare event. For the previously mentioned parameter space, it is expected to occur with the rate $\lesssim 10^{-13}-10^{-9}$ with respect to the ordinary photon production rate. Hence, its observation presents a challenge for the detector design and performance. The experimental setup specifically designed to search for the $A^{\prime} \rightarrow e^{+} e^{-}$decays is schematically shown in Fig. 1] The experiment could employ, e.g. the CERN SPS H4 $e^{-}$ beam, which is produced in the target T2 of the CERN 


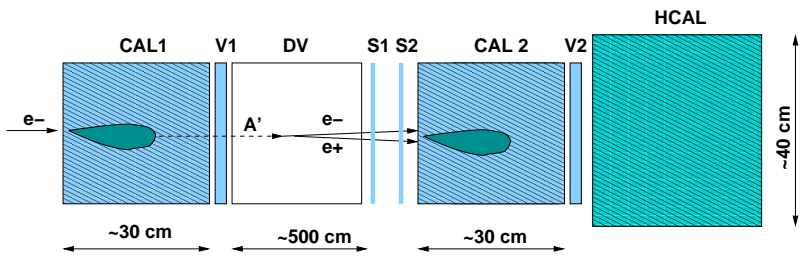

FIG. 1: Schematic illustration of the setup to search for dark photons in a light-shining-through-a-wall-type experiment at high energies. The incident electron energy absorption in the calorimeter CAL1 is accompanied by the emission of bremsstrahlung $A^{\prime}$ s in the reaction $e Z \rightarrow e Z A^{\prime}$ of electrons scattering on nuclei, due to the $\gamma-A^{\prime}$ mixing, as shown in Fig. 2. The part of the primary beam energy is deposited in the CAL1, while the rest of the total energy is transmitted by the $A^{\prime}$ through the CAL1 wall. The $A^{\prime}$ penetrates the CAL1 and veto $\mathrm{V} 1$ without interactions and decays in flight in the DV into a narrow $e^{+} e^{-}$pair, which generates the second electromagnetic shower in the CAL2 resulting in the two-shower signature in the detector. The sum of energies deposited in the CAL1+CAL2 is equal to the primary beam energy.

SPS and transported to the detector in an evacuated beam line tuned to a freely adjustable beam momentum from 10 up to $300 \mathrm{GeV} / \mathrm{c}$ [4]. The typical maximal beam intensity at $\simeq 30-50 \mathrm{GeV}$, is of the order of $\simeq 10^{6} e^{-}$for one typical SPS spill with $10^{12}$ protons on target. The typical SPS cycle for a fixed target (FT) operation lasts $14.8 \mathrm{~s}$, including $4.8 \mathrm{~s}$ spill duration. The maximal number of FT cycles is four per minute. The admixture of the other charged particles in the beam (beam purity) is below $10^{-2}$, and the size of the beam at CAL1 is of the order of a few $\mathrm{cm}^{2}$.

The detector shown in Fig 1 is equipped with a high density, compact electromagnetic (e-m) CAL1 to detect $e^{-}$primary interactions, high efficiency veto counters V1 and V2, two scintillating fiber counters (or proportional chambers) S1, S2 an electromagnetic calorimeter CAL2 located at the downstream end of the $A^{\prime}$ decay volume (DV) to detect $e^{+} e^{-}$pairs from $A^{\prime} \rightarrow e^{+} e^{-}$decays in flight, and a hadronic calorimeter (HCAL) used mainly for the $A^{\prime} \rightarrow$ invisible decay mode (see Sec.V). For searches at low energies the DV could be replaced by a Cherenkov counter to enhance the decay electrons tagging.

The method of the search is the following. The $A^{\prime} \mathrm{s}$ are produced through the mixing with bremsstrahlung photons from the electrons scattering off nuclei in the CAL1,

$$
\begin{gathered}
e^{-} Z \rightarrow e^{-} Z A^{\prime} \\
A^{\prime} \rightarrow e^{+} e^{-},
\end{gathered}
$$

as shown in Fig. 22 The reaction (2) is typically occurred at a few first radiation lengths $\left(X_{0}\right)$ of the detector. The bremsstrahlung $A^{\prime}$ then penetrates the rest of the CAL1 and the veto counter V1 without interactions, and decays in flight into an $e^{+} e^{-}$pair in the DV. A fraction $(f)$ of the primary beam energy $E_{1}=f E_{0}$ is deposited in the
CAL1. The CAL1's downstream part serves as a dump to absorb completely the e-m shower tail. For the radiation length $X_{0} \lesssim 1 \mathrm{~cm}$, and the total thickness of the CAL1 $\simeq 30 \mathrm{~cm}$ the energy leak from the CAL1 into the V1 is negligibly small. The remaining part of the primary electron energy $E_{2}=(1-f) E_{0}$ is transmitted trough the "CAL1 wall" by the $A^{\prime}$, and deposited in the second downstream CAL2 via the $A^{\prime}$ decay in flight in the DV, as shown in Fig1 At high $A^{\prime}$ energies $E_{A^{\prime}} \gtrsim 100 \mathrm{GeV}$, the opening angle $\Theta_{e^{+} e^{-}} \simeq M_{A^{\prime}} / E_{A^{\prime}}$ of the decay $e^{+} e^{-}$ pair is too small to be resolved in two separated tracks in the $\mathrm{S} 1$ and $\mathrm{S} 2$, or in two e-m showers in the CAL2, so the pairs are mostly detected as a single track or e-m shower.

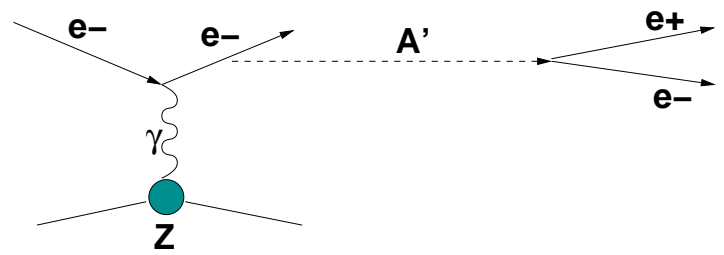

FIG. 2: Diagram illustrating the massive $A^{\prime}$ production in the reaction $e^{-} Z \rightarrow e^{-} Z A^{\prime}$ of electrons scattering off a nuclei $(A, Z)$ with the subsequent $A^{\prime}$ decay into an $e^{+} e^{-}$pair.

The occurrence of $A^{\prime} \rightarrow e^{+} e^{-}$decays produced in $e^{-} Z$ interactions would appear as an excess of events with two e-m-like showers in the detector, one shower in the CAL1 and another one in the CAL2, as shown in Fig 1, above those expected from the background sources. The signal candidate events have the signature:

$$
S_{A^{\prime}}=\mathrm{CAL} 1 \cdot \overline{\mathrm{V} 1} \cdot \mathrm{S} 1 \cdot \mathrm{S} 2 \cdot \mathrm{CAL} 2 \cdot \overline{\mathrm{V} 2} \cdot \overline{\mathrm{HCAL}}
$$

and should satisfy the following selection criteria:

- the starting point of (e-m) showers in the CAL1 and CAL2 should be localized within a few first $X^{0} \mathrm{~s}$.

- the lateral and longitudinal shapes of both showers in the CAL1 and CAL2 are consistent with an electromagnetic one. The fraction of the total energy deposition in the CAL1 is $f \lesssim 0.1$, while in the CAL2 it is $(1-f) \gtrsim 0.9$ (see Fig. 2 and discussion below).

- no energy deposition in the V1 and V2.

- the signal (number of photoelectrons) in the decay counters S1 and S2 is consistent with the one expected from two minimum ionizing particle (mip) tracks. At low beam energies, $E_{0} \lesssim 30 \mathrm{GeV}$, two isolated hits in each counter are requested.

- the sum of energies deposited in the CAL1+CAL2 is equal to the primary energy, $E_{1}+E_{2}=E_{0}$. 


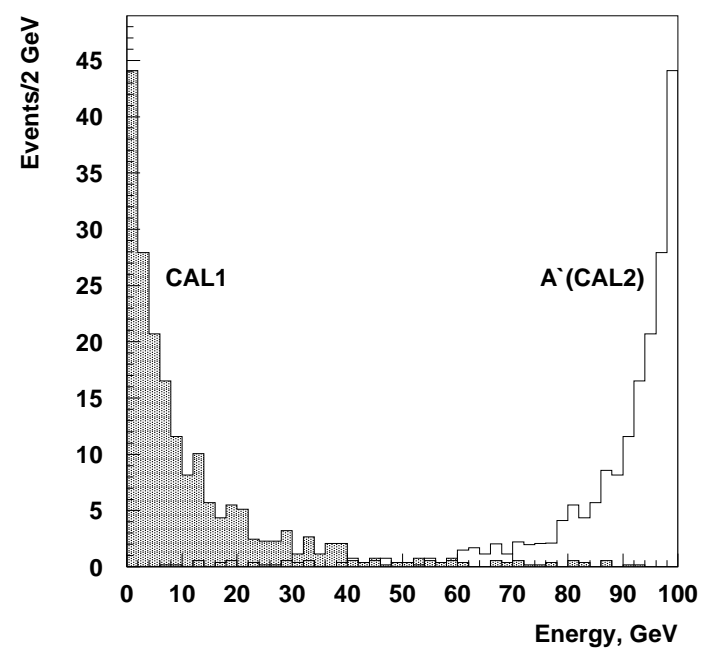

FIG. 3: Expected distributions of energy deposition for selected events: (i) in the CAL1(shaded), and (ii) in the CAL2 from the bremsstrahlung $A^{\prime} \rightarrow e^{+} e^{-}$s decays in flight in the DV region. The spectra are calculated for the $10 \mathrm{MeV} A^{\prime}$ s produced by $100 \mathrm{GeV}^{-}$'s in the CAL1 with momentum pointing towards the CAL2 fiducial area and the mixing strength $\epsilon=3 \cdot 10^{-4}$. For this mixing value, most of the $A^{\prime}$ s decay outside of the CAL1 in the DV. The distributions are normalized to a common maximum.

To estimate the sensitivity of the proposed experiment a simplified feasibility study based on Geant4 [5] Monte Carlo simulations have been performed for 10 and 300 $\mathrm{GeV}$ electrons. The CAL1 and CAL2 are the hodoscope arrays of the lead tungstate (PWO) heavy crystal counters $\left(X_{0} \simeq 0.89 \mathrm{~cm}\right)$, each of the size $10 \times 10 \times 300 \mathrm{~mm}^{3}$, allowing accurate measurements of the lateral and longitudinal shower shape. The veto counters are assumed to be 1-2 cm thick, high sensitivity LYSO crystal arrays with a high light yield of $\simeq 10^{3}$ photoelectrons per $1 \mathrm{MeV}$ of deposited energy. It is also assumed that the veto's inefficiency for the mip detection is, conservatively, $\lesssim 10^{-4}$. Each of the decay counters S1 and S2 consists of two layers of scintillating fiber strips, arranged, respectively, in the $\mathrm{X}$ and $\mathrm{Y}$ directions. Each strip consists of about 100 fibers of $1 \mathrm{~mm}$ square. The number of photoelectrons produced by a mip crossing the strip is $\simeq$. The energy resolution of the CAL1 and CAL2 calorimeters as a function of the beam energy is taken to be $\frac{\sigma}{E}=\frac{2.8 \%}{\sqrt{E}} \oplus 0.4 \% \oplus \frac{142 \mathrm{MeV}}{E}[\underline{6}]$. The energy threshold in the CAL1 is $0.5 \mathrm{GeV}$. The reported further analysis also takes into account passive materials from the DV tank walls.

The total number of $A^{\prime}$ s produced by $n_{e}$ electrons impinging a target with thickness $t \gg X_{0}$ is [7]:

$$
n_{A^{\prime}} \sim n_{e} C \frac{\epsilon^{2} m_{e}^{2}}{M_{A^{\prime}}^{2}}
$$

where parameter $C \simeq 10$ is only logarithmically depen- dent on the choice of target nucleus, and $m_{e}$ is the electron mass, for recent works on heavy particles production through photon exchange with a nucleus, see, also, Refs. [8, 9]. One can see that compared to the bremsstrahlung rate, the $A^{\prime}$ production rate is suppressed by a factor $\simeq \epsilon^{2} m_{e}^{2} / M_{A^{\prime}}^{2}$. The $A^{\prime}$ energy spectrum is [7]

$$
\frac{d n_{A^{\prime}}}{d E_{A^{\prime}}} \sim k \cdot x\left(1+\frac{x^{2}}{3(1-x)}\right)
$$

where $k$ is a constant, and $x=E_{A^{\prime}} / E_{0}$. In Fig. 3, an example of the expected distributions of energy deposition in the CAL1 and CAL2 for selected events are shown for the initial $e^{-}$energy of $100 \mathrm{GeV}$. The spectra are calculated for the mixing strength $\epsilon=3 \times 10^{-4}$ and corresponds to the case when the $A^{\prime}$ decay pass length $L_{A^{\prime}}$ is in the range $L^{\prime}<L_{A^{\prime}}<L$, where $L^{\prime}$ is the length of the CAL1, and $L$ is the distance between the $A^{\prime}$ production vertex and the CAL2. In this case most of $A^{\prime} \mathrm{s}$ decay outside of the CAL1 in the DV. One can see, that the $A^{\prime}$ bremsstrahlung distribution is peaked at maximal beam energy.

\section{A. Background}

The background processes for the $A^{\prime} \rightarrow e^{+} e^{-}$decay signature $S_{A^{\prime}}$ of (3) can be classified as being due to physical- and beam-related sources. To perform full detector simulation in order to investigate these backgrounds down to the level $\lesssim 10^{-12}$ would require a huge number of generated events resulting in a prohibitively large amount of computer time. Consequently, only the following, identified as the most dangerous processes are considered and evaluated with reasonable statistics combined with numerical calculations:

- the leak of the primary electron energy into the CAL2, could be due to the bremsstrahlung process $e^{-} Z \rightarrow e^{-} Z \gamma$, when the emitted photon carries away almost all initial energy, while the final state electron with the much lower energy $E_{e^{-}} \simeq 0.1 E_{0}$ is absorbed in the CAL1. The photon could punch through the CAL1 and V1 without interactions, and produce an $e^{+} e^{-}$pair in the $\mathrm{S} 1$, which deposits all its energy in the CAL2. The photon could also be absorbed in a photonuclear reaction $\gamma W \rightarrow \pi^{ \pm} X$ in the CAL1 resulting in, e.g. an energetic leading secondary pion or neutron accompanied by a small hadronic activity in the CAL1.

In the first case, to suppress this background, one has to use the CAL1 of enough thickness, and as low a veto threshold as possible. Taking into account that the primary interaction vertex is selected to be within the few first $X_{0}$ 's and the probability for the bremsstrahlung photon to carry away $\gtrsim 90 \%$ of the primary electron energy $\simeq 10^{-2}$, for the total remaining CAL1+V1 thickness of $\simeq 30 X_{0}$, the probability for the photon to punch through it without 
interaction per impinging electron is $\lesssim 10^{-12}$. Assuming that the photon conversion probability in $\mathrm{S} 1$ is $2 \times 10^{-2}$, this background is expected to be at the negligible level $\lesssim 2 \cdot 10^{-14}$. In the second case, the analysis results in a similar background level $\lesssim 10^{-13}$, mainly due to a small probability for secondary hadron to carry away almost all beam energy. Thus, the requirement to have low energy in the CAL1, and almost all beam energy deposited in the CAL2, is crucial for the background rejection of this type. If, for example, events are selected with the fraction of total energy deposited in the CAL1 $f \lesssim 0.3$, instead of $f \lesssim 0.1$, the signal-tobackground ratio drops by a factor $\simeq 10$, while the signal efficiency is increased just by $\simeq 20 \%$.

- punch-through primary electrons, which penetrate the CAL1 and V1 without depositing much energy, could produce a fake signal event. It is found that this is also an extremely rare event.

The beam-related background can be categorized as being due to a beam particle misidentified as an electron. This background is caused by some pion, proton and muon contamination in the electron beam.

- the first source of this type of background is due to the

$$
p(\pi)+A \rightarrow n+\pi^{0}+X, n \rightarrow \mathrm{CAL} 2
$$

reaction chain: (i) an incident proton (or a pion) produces a neutral pion with the energy $E_{\pi^{0}} \lesssim$ $0.1 E_{0}$ and an energetic leading neutron carrying the rest of the primary collision with the nucleus $(A, Z)$, (ii) the neutral pion decays into photons which generate e-m shower in the CAL1, while (iii) the neutron penetrates the rest of the CAL1 and the V1 without interactions, scatters in the S1, producing low-energy secondaries and deposits all its energy in the CAL2. The probability for such chain reactions to occur can be estimated as

$$
P \simeq P_{p(\pi)} \cdot P_{\pi^{0} n} \cdot P_{S 1} \cdot P_{n}
$$

where $P_{p(\pi)}, P_{\pi^{0} n}, P_{S 1}, P_{n}$ are, respectively, the level of the admixture of hadrons, $P_{p(\pi)} \lesssim 10^{-2}$, the probability for the incoming hadron to produce the $\pi^{0} n$ pair in the CAL1, $P_{\pi^{0} n} \simeq 10^{-4}$, the probability for the leading neutron to interact in S1, $P_{S 1} \simeq$ $10^{-3}$, and the probability for the leading neutron to deposit all its energy in the CAL2, $P_{n} \simeq 10^{-3}$. This results in $P \lesssim 10^{-12}$. The probability for the neutron to interact in the $\mathrm{S} 1$ of thickness $\simeq 1 \mathrm{~mm}$, or $\simeq 10^{-3}$ nuclear interaction length can be reduced significantly, down to $P_{S 1} \simeq 10^{-4}$, by replacing it, e.g. with a thin wire chamber counter. This leads to $P \lesssim 10^{-13}$. At low energies $E_{0} \lesssim 30 \mathrm{GeV}$, the requirement to have two hits in the S1 would significantly suppress the background further.
Note that the total cross section for the reaction $p(\pi)+A \rightarrow \pi^{0}+n+X$ with the leading neutron in the final state has not yet been studied in detail for the wide class of nuclei and full range of hadron energies. To perform an estimate of the $P_{\pi^{0} n}$ value, we use available data from the ISR experiment at CERN, which measured leading neutron production in $p p$ collisions at $\sqrt{s}$ in the range of 20 to $60 \mathrm{GeV}$ [10, 11]. For these energies, the invariant cross sections, measured as a function of $x_{F}$ (Feynman $x$ ) and $p_{T}$, were found to be in the range $0.1 \lesssim E \frac{d^{3} \sigma}{d^{3} p} \lesssim 10 \mathrm{mb} / \mathrm{GeV}^{2}$ for $0.9 \lesssim x_{F} \lesssim 1$ and $0 \lesssim p_{T} \lesssim 0.6 \mathrm{GeV}$ [10]. Taking this into account, the cross sections for leading neutron production in our energy range are evaluated by using the Bourquin-Gaillard formula, which gives the parametric form of the invariant cross section for the production in high-energy hadronic collisions of many different hadrons over the full phase space, for more details see, e.g., Ref. [12]. The total leading neutron production cross sections in $p(\pi) A$ collisions are calculated from its linear extrapolation to the target atomic number.

In another scenario, the leading neutron could interact in the very last downstream part of the veto counter producing leading $\pi^{0}$ without being detected. The neutral pion decays subsequently into $2 \gamma$ or $e^{+} e^{-} \gamma$. The background from from this event's chain is also found to be very small.

- the fake signature $S_{A^{\prime}}$ arises when the incoming pion produces a low-energy neutral pion in the very beginning of the CAL1, escapes detection in the V1 due to its inefficiency, and either deposits all its energy in the CAL2, or decays in flight in the DV into an $e \nu$ pair with the subsequent electron energy deposition in the CAL2. In the first case, also relevant to protons, considerations similar to the previous one show that this background is expected to be at the level $\lesssim 10^{-13}$. In the second case, taking into account the probability for the $\pi \rightarrow e \nu$ decay in flight and the fact that the decay electron would typically have about one half of the pion energy, results in suppression of this background to the level $<10^{-15}$.

- another type of background is caused by the muon contamination in the beam. The muon could produce a low-energy photon in the CAL1, which would be absorbed in the detector, then penetrates the V1 without being detected, and after producing signals in the S1 and S2 deposits all its energy in the CAL2 through the emission of a hard bremsstrahlung photon:

$$
\mu+Z \rightarrow \gamma+\mu+Z, \mu \rightarrow \mathrm{CAL} 2
$$

The probability for the events chain (8) is estimated to be $P \lesssim 10^{-14}$. Similar to (6), this estimate is 
TABLE I: Expected contributions to the total level of background from different background sources ( see text for details).

\begin{tabular}{lr}
\hline \hline Source of background & Expected level \\
\hline punchthrough $e^{- \text {'s } \text { or } \gamma \text { 's }}$ & $\lesssim 10^{-13}$ \\
hadronic reactions & $\lesssim 2 \times 10^{-13}$ \\
$\mu$ reactions & $\lesssim 10^{-14}$ \\
accidentals & $\lesssim 10^{-14}$ \\
\hline Total ( conservative) & $\lesssim 3 \times 10^{-13}$ \\
\hline \hline
\end{tabular}

obtained assuming that the muon contamination in the beam is $\lesssim 10^{-2}$, the probability for the muon to cross the $\mathrm{V} 1$ without being detected is $\lesssim 10^{-4}$, and the probability for the $\mu$ to deposit all its energy in the CAL2 is $\lesssim 10^{-7}$. Here, it is also taking into account that the muon should stop in the CAL2 completely to avoid being detected in the veto V2. The additional suppression factor is due to the requirement to have two mip-like signals in the decay counters.

- one more background can be due the event chain

$$
\mu+Z \rightarrow \mu+\gamma+Z, \mu \rightarrow e \nu \nu
$$

when the incident muon produces in the initial CAL1 part a low-energy bremsstrahlung photon, escapes detection in the $\mathrm{V} 1$, and then decays in flight in the DV into $e \nu \nu$. There are several suppression factors for this source of background: (i) the relatively long muon lifetime resulting in a small probability to decay, and (ii) the presence of two neutrinos in the $\mu$ decay. The decay electron energy deposition in the CAL2 is typically significantly smaller than the primary energy $E_{0}$ and (iii) the requirement to have double mip energy deposition in the beam counters $\mathrm{S} 1$ and $\mathrm{S} 2$. All these factors lead to the expectation for this background to be at the level at least $\lesssim 10^{-14}$.

- a random superpositions of uncorrelated events occurring during the detector gate time could also result in a fake signal. However, taking into account the selection criteria of signal events and the fact that the beam time intensity profile is flat during the spill duration results in a small number of these background events $\lesssim 10^{-14}$.

In Table I contributions from the all background processes are summarized. The total background is conservatively at the level $\lesssim 3 \cdot 10^{-13}$, and is dominated by the admixture of hadrons in the electron beam. This means that the search accumulated up to $\simeq 10^{13} e^{-}$events, is expected to be background free. To evaluate background in the signal region one could perform independent direct measurements of its level with the same setup by using pion and muon beams of proper energies.

\section{B. Expected sensitivity}

The significance of the $A^{\prime} \rightarrow e^{+} e^{-}$decay discovery with such a detector, scales as [13, 14]

$$
S=2 \cdot\left(\sqrt{n_{A^{\prime}}+n_{b}}-\sqrt{n_{b}}\right)
$$

where $n_{A^{\prime}}$ is the number of observed signal events (or the upper limit of the observed number of events), and $n_{b}$ is the number of background events.

For a given number of $e^{-}$'s on the target (CAL1) of length $L^{\prime}, n_{e} \cdot t$ (here, $n_{e}$ is the electron beam intensity and $t$ is the experiment running time) and $A^{\prime}$ flux $d n_{A^{\prime}} / d E_{A^{\prime}}$, the expected number of $A^{\prime} \rightarrow e^{+} e^{-}$decays occurring within the fiducial volume of the DV with the subsequent energy deposition in the CAL2, located at a distance $L$ from the $A^{\prime}$ production vertex is given by

$$
\begin{array}{r}
n_{A^{\prime}} \sim n_{e} t \int A \frac{d n_{A^{\prime}}}{d E_{A^{\prime}}} \exp \left(-\frac{L^{\prime} M_{A^{\prime}}}{p_{A^{\prime}} \tau_{A}^{\prime}}\right) \\
{\left[1-\exp \left(-\frac{L M_{A^{\prime}}}{p_{A^{\prime}} \tau_{A}^{\prime}}\right)\right] \frac{\Gamma_{e^{+} e^{-}}}{\Gamma_{t o t}} \varepsilon_{e^{+} e^{-}} d E_{A^{\prime}} d V}
\end{array}
$$

where $p_{A^{\prime}}$ is the $A^{\prime}$ momentum, $\tau_{A^{\prime}}$ is its lifetime at the rest frame, $\Gamma_{e^{+} e^{-}}, \Gamma_{\text {tot }}$ are the partial and total $A^{\prime}$-decay widths, respectively, and $\varepsilon_{e^{+} e^{-}}(\simeq 0.9)$ is the $e^{+} e^{-}$pair reconstruction efficiency. The flux of $A^{\prime}$ s produced in reaction (2) is calculated by using the $A^{\prime}$ production cross section in the $e^{-Z}$ collisions from Ref. [7] (an example of the flux calculation is shown in Fig. 3). The acceptance $A$ of the CAL2 detector is calculated tracing $A^{\prime}$ s produced in the CAL1 to the CAL2. The corresponding $A^{\prime} \rightarrow e^{+} e^{-}$decay rate is given by

$$
\Gamma\left(A^{\prime} \rightarrow e^{+} e^{-}\right)=\frac{\alpha}{3} \epsilon^{2} M_{A^{\prime}} \sqrt{1-\frac{4 m_{e}^{2}}{M_{A^{\prime}}^{2}}}\left(1+\frac{2 m_{e}^{2}}{M_{A^{\prime}}^{2}}\right)
$$

It is assumed that this decay mode is dominant and the branching ratio $\frac{\Gamma\left(A^{\prime} \rightarrow e^{+} e^{-}\right)}{\Gamma_{t o t}} \simeq 1$.

If no excess events are found, the obtained results can be used to impose bounds on the $\gamma-A^{\prime}$ mixing strength as a function of the dark photon mass. Taking Eqs.10 - 12) into account and using the relation $n_{A^{\prime}}\left(M_{A^{\prime}}\right)<n_{A^{\prime}}^{90 \%}\left(M_{A^{\prime}}\right)$, where $n_{A^{\prime}}^{90 \%}\left(M_{A^{\prime}}\right)$ is the $90 \%$ C.L. upper limit for the number of signal events from the decays of the $A^{\prime}$ with a given mass $M_{A^{\prime}}$ one can determine the expected $90 \%$ C.L. exclusion area in the $\left(M_{A^{\prime}} ; \epsilon\right)$ plane from the results of the experiment. For the background-free case $\left(n_{A^{\prime}}^{90 \%}\left(M_{A^{\prime}}\right)=2.3\right.$ events $)$, the exclusion regions corresponding to accumulated statistics

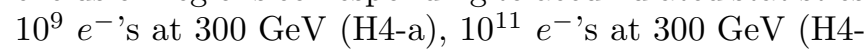

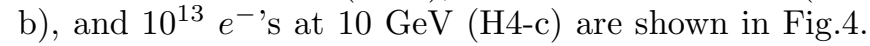
One can see, that these exclusion areas are complementary to the ones expected from the planned APEX (full run) and DarkLight experiments, which are also shown for comparison [2]. For a review of all experiments, which intend to probe a similar parameter space, see Ref. [2] and references therein. Shown also are areas excluded from 


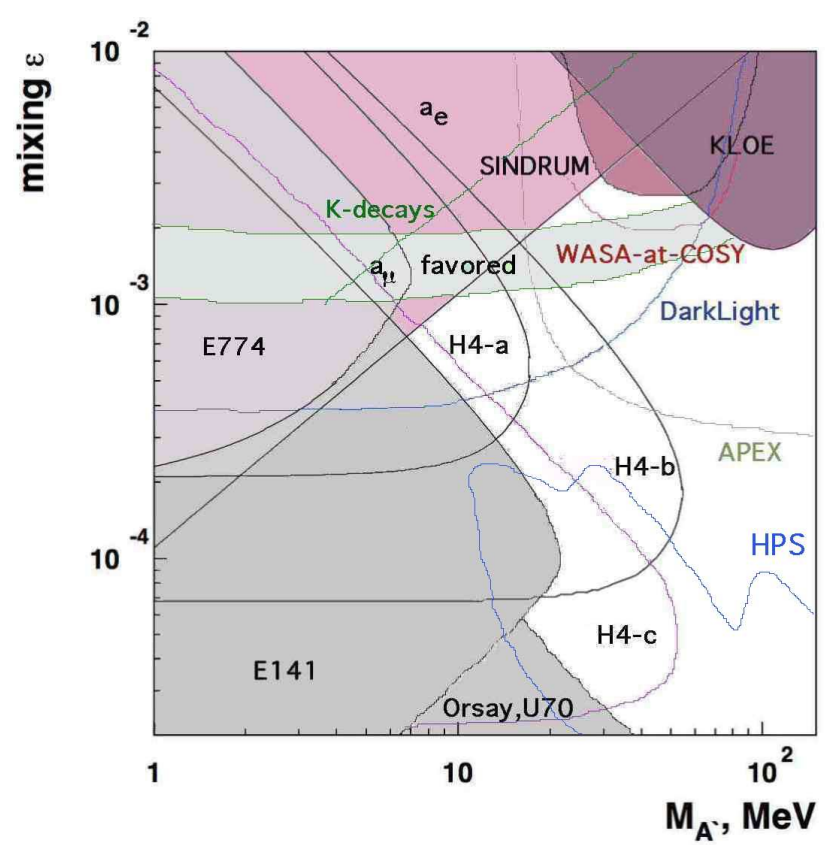

FIG. 4: Exclusion region in the $\left(M_{A^{\prime}} ; \epsilon\right)$ plane obtained in the present work from the expected results of the experiments accumulated $10^{9} e^{-}$'s at $300 \mathrm{GeV}$ (H4-a), $10^{11} e^{-}$'s

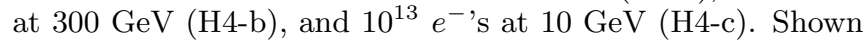
are also areas excluded from the electron (g-2) considerations $\left(a_{e}\right)$ [15], by the results of the electron beam-dump experiments E141 [16] and E774 [17], by searches at LAL Orsay 18], U70 (Protvino) [19], and KLOE [20], from kaon decays 21] and data of the experiment SINDRUM [22, 23], and by the WASA-at-COSY Collaboration 24]. Expected sensitivities of the planned APEX (full run), HPS and DarkLight experiments are also shown for comparison [2]. For a review of all experiments, which intend to probe a similar parameter space, see Ref. 2] and references therein. In addition the light grey area shows the $\pm 2 \sigma$ preferred band from the muon g- 2 anomaly consideration.

the electron $(\mathrm{g}-2)$ considerations $\left(a_{e}\right)$ [15], by the results of the electron beam-dump experiments E141 [16] and E774 [17], by searches at LAL Orsay [18], U70 (Protvino) [19], and KLOE [20], from kaon decays [21] and data of the experiment SINDRUM [22, 23], and by the WASA-atCOSY Collaboration [24]. For cosmological constraints on dark matter particles charged under a hidden gauge group, see, e.g. [25].

The statistical limit on the sensitivity of the proposed experiment is set mostly by the value of mixing strength. Thus, to accumulate large number of events is important. As one can see from Eq.(11), the obtained exclusion regions are also sensitive to the choice of the length $L^{\prime}$ of the CAL1, which should be as short as possible. Assuming the average $\mathrm{H} 4$ beam rate $n_{e} \gtrsim 10^{5} e^{-} / \mathrm{s}$ at $E_{0} \simeq 200-300 \mathrm{GeV}$, we anticipate $\simeq 3 \times 10^{11} e^{-9} \mathrm{~s}$ on CAL1 during $\simeq 1$ month of running time for the experiment. At lower energies the $e^{-}$beam intensity is increased and much higher statistics can be accumulated. Note, however, that since the decay time of the PWO/LYSO light signal is $\tau \lesssim 50 \mathrm{~ns}$, the maximally allowed electron counting rate, has to be $\lesssim 1 / \tau \simeq 10^{7} e^{-} / \mathrm{s}$ to avoid significant pile-up effect. To minimize dead time, one could use a first-level trigger rejecting events with the CAL2 energy deposition less than, say, the energy $\simeq 0.9 E_{0}$ and, hence, run the experiment at a higher rate.

In the case of the signal observation, to cross-check the result, one could remove the DV and put the CAL2 behind the CAL1. This would not affect the main background sources and still allow the $A^{\prime}$ 's production, but with their decays in front of the CAL2 being suppressed. In this case the distribution of the energy deposition in the CAL1 and CAl2 would contain mainly background events, while the signal from the decays $A^{\prime} \rightarrow e^{+} e^{-}$should be reduced. The background can also be independently studied with a high-energy muon and pion beams. The evaluation of the $A^{\prime}$ mass could be obtained from the results of measurements at different distances $L$ and beam energies. Finally note, that the performed analysis gives an illustrative order of magnitude for the sensitivity of the proposed experiment and may be strengthened by more accurate and detailed simulations of the $\mathrm{H} 4$ beam-line and experimental setup.

\section{THE EXPERIMENT TO SEARCH FOR THE DECAY $A^{\prime} \rightarrow$ invisible}

The $A^{\prime}$ s could also decay invisibly into a pair of dark matter particles $\chi \bar{\chi}$, see Refs.[26, 27] and references therein. The process of the dark photon production and

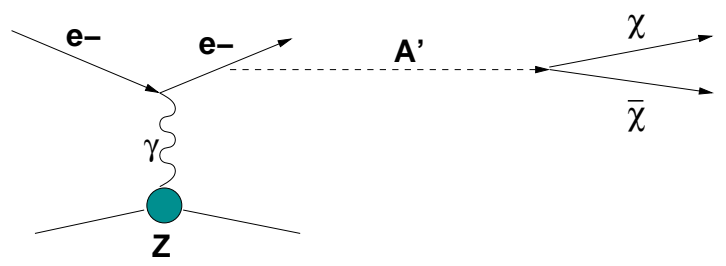

FIG. 5: Diagram illustrating the massive $A^{\prime}$ production in the reaction $e^{-} Z \rightarrow e^{-} Z A^{\prime}$ of electrons scattering off a nuclei $(\mathrm{A}, \mathrm{Z})$ with the subsequent $A^{\prime}$ decay into a $\chi \bar{\chi}$ pair.

subsequent invisible decay

$$
\begin{gathered}
e^{-} Z \rightarrow e^{-} Z A^{\prime} \\
A^{\prime} \rightarrow \text { invisible }
\end{gathered}
$$

shown in Fig. 5 is expected to be very rare event. For the previously mentioned parameter space, it is expected to occur with the rate $\lesssim 10^{-10}$ with respect to the ordinary photon production rate. Hence, its observation presents a challenge for the detector design and performance. 


\section{A. The setup}

The detector specifically designed to search for the $A^{\prime} \rightarrow$ invisible decays is schematically shown in Fig. 1 . The experiment employs the same very clean high-energy $e^{-}$beam for the search for the $A^{\prime} \rightarrow e^{+} e^{-}$decays. The detector shown in Fig. 1 is additionally equipped with a massive HCAL, located at the downstream end of the setup to detect all final state products from the primary reaction $e^{-Z} \rightarrow$ anything (see below).

The method of the search is the following. The $A^{\prime} \mathrm{s}$ are produced through the mixing with bremsstrahlung photons from the electrons scattering off nuclei in the CAL1. The reaction (13) typically occurs in the first few radiation lengths of the detector. The bremsstrahlung $A^{\prime}$ then penetrates the rest of the setup without interactions and decays in flight invisibly, $A^{\prime} \rightarrow$ invisible, into a pair of dark matter particles, which also penetrate the rest of the setup without interaction. Similar to the previous case, the fraction $f$ of the primary beam energy $E_{1}=f E_{0}$ is deposited in the CAL1 by the scattered electron. The CAL1's downstream part serves as a dump to absorb completely the e-m shower tail. For the total thickness of the CAL1 $\simeq 30 X_{0}$, the energy leak from the CAL1 into the V1 is negligibly small. The remaining part of the primary electron energy $E_{2}=(1-f) E_{0}$ is carried away by the products of the decay $A^{\prime} \rightarrow \chi \bar{\chi}$. In order to suppress background due to the detection inefficiency, the detector must be longitudinally completely hermetic. To enhance detector hermeticity, the hadronic calorimeter with a total thickness $\simeq 20 \lambda_{\text {int }}$ (nuclear interaction lengths) is placed behind the CAL2, as shown in Fig. 1. Under the assumption that the $A^{\prime}$ decays dominantly into the invisible final state, the calorimeter CAL1 is not constrained in length anymore, as it was for the case of $A^{\prime} \rightarrow e^{+} e^{-}$decays. The CAL1(and CAL2) could be, e.g. a hodoscope array of the PWO crystal counters, or another e-m calorimeter of similar performance. The

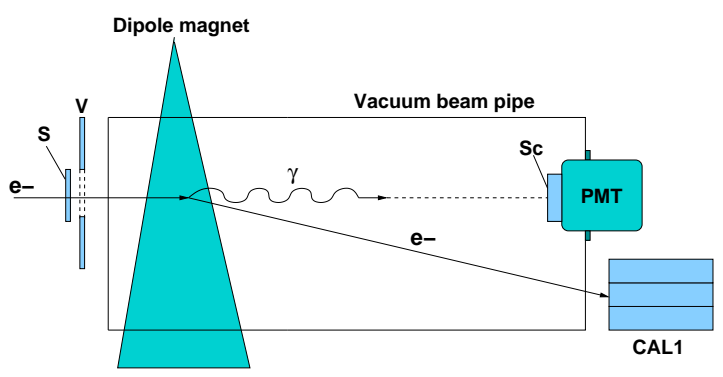

FIG. 6: The scheme of the additional tagging of high-energy electrons in the beam by using the electron synchrotron radiation in the banding magnetic dipole. The synchrotron radiation photons are detected by a $\gamma$ - detector by using the LYSO inorganic crystal (Sc) capable for the work in vacuum. The crystal is viewed by a high quantum efficiency photodetector, e.g. PMT, SiPM, or APD. The beam defining counters S and veto $\mathrm{V}$ are also shown. occurrence of $A^{\prime} \rightarrow$ invisible decays produced in $e^{-} Z$ interactions would appear as an excess of events with a single e-m shower in the CAL1, see Fig. 1, and zero energy deposition in the rest of the detector, above those expected from the background sources. The signal candidate events have the signature:

$$
S_{A^{\prime}}=\mathrm{CAL} 1 \cdot \overline{\mathrm{V} 1 \cdot \mathrm{S} 1 \cdot \mathrm{S} 2 \cdot \mathrm{CAL} 2 \cdot \mathrm{V} 2 \cdot \mathrm{HCAL}}
$$

and should satisfy the following selection criteria:

- The starting point of (e-m) showers in the CAL1 should be localized within the few first $X_{0}$ s.

- The lateral and longitudinal shapes of the shower in the CAL1 are consistent with an electromagnetic one. The fraction of the total energy deposition in the CAL1 is $f \lesssim 0.1$, while in the CAL2, it is zero.

- No energy deposition in the V1, S1,S2, CAL2, V2, and HCAL.

\section{B. Background}

The background reactions resulting in the signature of Eq.refsigninv can be classified as being due to physicaland beam-related sources. Similar to the case of the decay $A^{\prime} \rightarrow e^{+} e^{-}$, to perform a full detector simulation in order to investigate these backgrounds down to the level $\lesssim 10^{-10}$ would require a prohibitively large amount of computer time. Consequently, only the following background sources, identified as the most dangerous are considered and evaluated with reasonable statistics combined with numerical calculations:

- One of the main background sources is related to the low-energy tail in the energy distribution of beam electrons. This tail is caused by the electron interactions with a passive material, such as entrance windows of the beam lines, residual gas, etc... Another source of low-energy electrons is due to the pion or muon decays in flight in the beam line. The uncertainties arising from the lack of knowledge of the dead material composition in the beam line are potentially the largest source of systematic uncertainty in accurate calculations of the fraction and energy distribution of these events. An estimation shows that the fraction of events with energy below $\lesssim 10 \mathrm{GeV}$ in the electron beam tuned to $100 \mathrm{GeV}$ could be as large as $10^{-8}$. Hence, the sensitivity of the experiment could be determined by the presence of such electrons in the beam, unless one takes special measures to suppress this background.

To improve the high-energy electrons selection and suppress background from the possible admixture of low-energy electrons, one can use a tagging system utilizing the synchrotron radiation (SR) from 
high-energy electrons in a dipole magnet, installed upstream of the detector, as schematically shown in Fig. 6. The basic idea is that, since the critical SR photon energy is $(\hbar \omega)_{\gamma}^{c} \propto E_{0}^{3}$, the low-energy electrons in the beam could be rejected by using the cut, e.g. $E_{\gamma}>0.3(\hbar \omega)_{\gamma}^{c}$, on the energy deposited in an X-ray detector shown in Fig. 6. For detection of the SR photons in vacuum one can utilize the inorganic LYSO crystal with a high light yield. The possibility of identifying electrons by detecting their synchrotron radiation has been demonstrated previously, see, e.g., Ref. 28]. Note that additionally, electrons with energy $\lesssim 10 \mathrm{GeV}$ will be deflected by the magnet at an angle which is larger than those for $100 \mathrm{GeV} e^{-}$, and, hence do not hit the CAL1. However, low-energy electrons could appear in the beam after the magnet due to the muon $\mu \rightarrow e \nu \nu$ or pion $\pi \rightarrow e \nu$ decays in flight. Since $\mu$ s and $\pi \mathrm{s}$ do not emit SR photons with energy above the cut, this source of background will also be suppressed.

- The fake signature of Eq. (14) could also arise when either (i) a beam hadron produces a low energy neutral pion in the very beginning of the CAL1 and then escapes detection in the rest of the detector, or (ii) a leading hadron $h$ from the reaction $e A \rightarrow e h X$ occurring in a very upstream part of the CAL1 is not detected. In the first case, the background is suppressed by the requirement of the presence of the synchrotron photon in the beam line. In the second case, background is dominated by the incomplete hermeticity of the detector. The leak of energy could be due to the production of a leading neutral hadron, such as a neutron and/or $K_{L}^{0}$, which punch through the CAL2 and HCAL without depositing energy above a certain threshold $E_{t h}$. An event with the sum of energy released in the CAL2 and HCAL below $E_{t h}$ is considered as "zero-energy" event. The punchthrough probability is defined by $\exp \left(-L_{t o t} / \lambda_{\text {int }}\right)$, where $L_{t o t}$ is the (CAL2+HCAL) sum length. It is of the order $10^{-9}$ for the total thickness of the CAL2 and HCAL about $21 \lambda_{\text {int }}$. This value should be multiplied by a conservative factor $\lesssim 10^{-4}$, which is the probability of a single leading hadron photo- or electroproduction in the CAL1. Taking this into account results in the final estimate of $\lesssim 10^{-13}$ for the level of this background per incoming electron.

- The HCAL nonhermeticity for high-energy hadrons was cross-checked with Geant4-based simulations. The low-energy tail in the distribution of energy deposited by $\simeq 10^{7}$ simulated $100 \mathrm{GeV}$ neutrons in the CAL2+HCAL was fitted by a smooth polynomial function and extrapolated to the lowest energy region in order to evaluate the number of events below a certain threshold $E_{t h}$. This procedure resulted in an estimate of the (CAL2+HCAL)- nonhermeticity, defined as the ratio of the number of events below the threshold $E_{t h}$ to the total number of incoming particles: $\eta=n\left(E<E_{t h}\right) / n_{t o t}$. For the energy threshold $E_{t h} \simeq 1 \mathrm{GeV}$ the nonhermeticity is expected to be at the level $\eta \lesssim 10^{-9}$. Taking into account the probability to produce the single leading hadron per incoming electron to be $P_{h} \lesssim 10^{-4}$, results in an overall level of this background of $\lesssim 10^{-13}$, in agreement with the previous rough estimate.

In Table I contributions from the all background processes are summarized for the beam energy of $100 \mathrm{GeV}$. The total background is conservatively at the level $\lesssim$ $10^{-12}$. This means that the search that accumulated up to $\simeq 10^{12} e^{-}$events is expected to be background free.

TABLE II: Expected contributions to the total level of background from different background sources estimated for the beam energy $100 \mathrm{GeV}$ (see text for details).

\begin{tabular}{lr}
\hline \hline Source of background & Expected level \\
\hline punchthrough $e^{-}$s or $\gamma \mathrm{S}$ & $\lesssim 10^{-13}$ \\
HCAL nonhermeticity & $\lesssim 10^{-13}$ \\
$e^{-}$low-energy tail, $E_{e} \lesssim 0.1 E_{0}$ & $\lesssim 10^{-13}$ \\
$\mu$ reactions & $\lesssim 10^{-13}$ \\
$e^{-}$-induced photo-nuclear reactions & $\lesssim 10^{-13}$ \\
\hline Total (conservative) & $\lesssim 5 \times 10^{-13}$ \\
\hline \hline
\end{tabular}

\section{Expected sensitivity}

Using considerations, which are similar to those of Sec.IIB, the expected exclusion areas in the plane $\left(\epsilon, M_{A^{\prime}}\right)$ derived for the background-free case are shown in Fig. 7 for accumulated statistics of $10^{9}$ (light blue) and $10^{12}$ (blue) $e^{-} \mathrm{s}$ with energy $100 \mathrm{GeV}$. The only assumption used is that the $A^{\prime}$ s decay dominantly to the invisible final state $\chi \bar{\chi}$, if the $A^{\prime}$ mass $M_{A^{\prime}}>2 m_{\chi}$, for more details, see Ref.[29]. Similar to the case of the visible $A^{\prime}$ decay search, the statistical limit on the sensitivity of the proposed experiment is proportional to $\epsilon^{2}$ and is mostly set by its value. Thus, it is important to accumulate a large number of events. In the case of the $A^{\prime} \rightarrow$ invisible signal observation, several methods could be used to cross-check the result. For instance, to test whether the signal is due to the HCAL nonhermeticity or not, one could perform several measurements with different HCAL thicknesses. In this case the expected background level can be obtained by extrapolating the results to a very large (infinite) HCAL thickness.

\section{CONCLUSION}

Due to their specific properties, dark photons are an interesting probe of physics beyond the standard model 


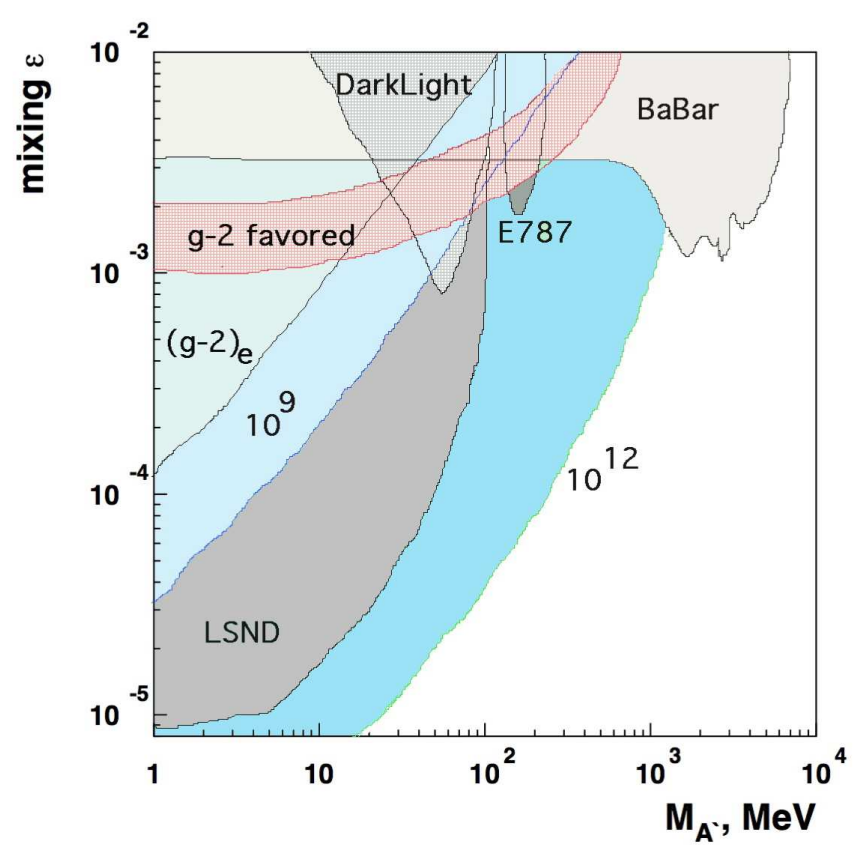

FIG. 7: Constraints in the $\epsilon$ vs $M_{A^{\prime}}$ plane for invisibly decaying $A^{\prime}$ into a pair of light dark-matter particles $\chi \bar{\chi}$, provided $M_{A^{\prime}}>2 m_{\chi}$. The light blue and blue areas show the expected $90 \%$ C.L. exclusion areas corresponding, respectively, to $10^{9}$ and $10^{12}$ accumulated electrons at $100 \mathrm{GeV}$ for the background-free case. Other existing constraints, mostly adapted from Ref. [27], are also shown. The constraint from the BaBar monophoton search is given as the light grey shaded region. Further limits are shown from the anomalous magnetic moment of the electron $\left((g-2)_{e}\right)$, and DarkLight, the rare kaon decay $K^{+} \rightarrow \pi^{+} A^{\prime}$ (E787), and LSD experiments. The LSND area is determined assuming $A^{\prime}-\chi$ coupling $\alpha_{D}=0.1$, and that $\chi$ cannot decay to other light dark-sector states which do not interact with $A^{\prime}$ s [30]. The red shaded region is preferred in order to explain the discrepancy between the measured and the predicted value of the anomalous magnetic moment of the muon. A more complete plot including various other constraints from performed and planned experiments can be found in Ref.[29].

both from the theoretical and experimental viewpoints. We proposed to perform a light-shining-through-a-wall experiment dedicated to the sensitive search for dark photons in the still unexplored area of the mixing strength $10^{-5} \lesssim \epsilon \lesssim 10^{-3}$ and masses $M_{A^{\prime}} \lesssim 100 \mathrm{MeV}$ by using available $10-300 \mathrm{GeV}$ electron beams from the CERN SPS. If $A^{\prime}$ s exist, their dielectron decays $A^{\prime} \rightarrow e^{+} e^{-}$ could be observed by looking for events with the twoshower topology of energy deposition in the detector. The key point for the experiment is an observation of events with almost all beam energy deposition in the CAL2, located behind the CAL1 wall. The advantage of the proposed search is that for the area of the mixing $10^{-4} \lesssim \epsilon \lesssim 10^{-3}$ and masses $10 \lesssim M_{A^{\prime}} \lesssim 100 \mathrm{MeV}$ its sensitivity is roughly proportional to the mixing squared, $\epsilon^{2}$, different from the case of a search for a long-lived $A^{\prime}$, where the number of signal events is $\propto \epsilon^{4}$.

A feasibility study of the experimental setup showed that the sensitivity of the search for the $A^{\prime} \rightarrow e^{+} e^{-}$decay in ratio of cross sections $\frac{\sigma\left(e^{-} Z \rightarrow e^{-} Z A^{\prime}\right)}{\sigma\left(e^{-} Z \rightarrow e^{-} Z \gamma\right)}$ at the level of $\lesssim 10^{-13}$ could be achieved. This sensitivity could be obtained with a setup optimized for several of its properties. Namely, (i) the intensity and purity of the primary electron beam, (ii) the high efficiency of the veto counters (iii) high number of photoelectrons from decays counters S1 and S2, iv) the good energy, time resolution and capability to measure accurately longitudinal and lateral shape of showers in both CAL1 and CAL2 calorimeters are of importance. A large amount of high energy electrons and high background suppression is crucial to improve the sensitivity of the search. To obtain the best sensitivity for a particular parameter region, the choice of the energy and intensity of the beam as well as the background level should be compromised. In the case of nonobservation, the expected exclusion areas are complementary to the ones from the planned APEX (full run), DarkLight, and other experiments intended to probe a similar parameter space [2].

The experiment has also the capability for a sensitive search for $A^{\prime}$ s decaying invisibly to dark-sector particles, such as dark matter. Our feasibility study showed that a sensitivity for the search of the $A^{\prime} \rightarrow$ invisible decay mode in branching fraction $\operatorname{Br}\left(A^{\prime}\right)=$ $\frac{\sigma\left(e^{-} Z \rightarrow e^{-} Z A^{\prime}\right), A^{\prime} \rightarrow \text { invisible }}{\sigma\left(e^{-} Z \rightarrow e^{-} Z \gamma\right)}$ at the level below a few parts in $10^{13}$ is in reach. The intrinsic background due to the presence of low-energy electrons in the beam can be suppressed by using a tagging system, which is based on the detection of synchrotron radiation of high energy electrons. The search would allow us to cover a significant fraction of the yet unexplored parameter space for the $A^{\prime} \rightarrow$ invisible decay mode.

This proposal provided interesting motivations for the search for light dark matter particles in order to perform it at CERN in the near future. The experiment might be a sensitive probe of new physics that is complementary to collider experiments. The required high-energy, intensity, and purity electron beams could also be available at future facilities such as the CLIC [31].

\section{Acknowledgments}

I would like to thank S. Andreas, P. Crivelli, S. Donskov, D. Gorbunov, M. Kirsanov, N. Krasnikov, L.Di Lella, V. Matveev, V. Polyakov, A. Radionov, A. Ringwald, A. Rubbia, V. Samoylenko, and K. Zioutas for useful discussions and comments and A. Fabich and L. Gatignon for valuable comments on the CERN SPS beam-lines. 
[1] J. Jaeckel and A. Ringwald, Annu. Rev. Nucl. Part. Sci. 60, 405 (2010).

[2] J.L. Hewett et al., arXiv:1205.2671,

[3] L.B. Okun, Zh. Eksp. Teor. Fiz. 83, 892 (1982)[Sov. Phys. JETP 56, 502 (1982)].

[4] See, for example, http://sba.web.cern.ch/sba/

[5] S. Agostinelli et al. (Geant4 Collaboration), Nucl. Instrum. Meth. A 506, 250 (2003); J. Allison et al. (Geant4 Collaboration) IEEE Trans. Nucl. Sc. 53, 270 (2006).

[6] P. Adzic et al. (CMS Collaboration), JINST 5, P03010 (2010).

[7] J. D. Bjorken, R. Essig, P. Schuster, and N. Toro, Phys. Rev. D 80, 075018 (2009).

[8] M. Masip, P. Masjuan, and D. Meloni, JHEP 1301, 106 (2013).

[9] A. Radionov, Phys. Rev. D 88, 015016 (2013).

[10] W. Flauger et al., Nucl. Phys. B 109, 347 (1976).

[11] J. Engler et al., Nucl. Phys. B 84, 70 (1975).

[12] S.N. Gninenko, Phys. Lett. B 713, 244 (2012).

[13] S.I. Bityukov and N.V. Krasnikov, Mod. Phys. Lett A 13, 3235 (1998).

[14] S.I. Bityukov and N.V. Krasnikov, Nucl. Instrum. Meth. A 534, 152 (2004).

[15] M. Endo, K. Hamaguchi, and G. Mishima, Phys.Rev. D 86, 095029 (2012).

[16] E. M. Riordan et al., Phys. Rev. Lett. 59, 755 (1987).

[17] A. Bross, M. Crisler, S. H. Pordes, J. Volk, S. Errede, and J. Wrbanek, Phys. Rev. Lett. 67, 2942 (1991).
[18] S. Andreas, C. Niebuhr, and A. Ringwald, Phys. Rev. D 86, 095019 (2012).

[19] J. Blümlein and J. Brunner, Phys. Lett. B 701, 155 (2011).

[20] F. Archilli et al., Phys. Lett. B 706, 251 (2012).

[21] T. Beranek and M. Vanderhaeghen, arXiv:1209.4561.

[22] S.N. Gninenko, Phys. Rev. D 87, 035030 (2013).

[23] R. Meijer Drees et al.(SINDRUM Collaboration), Phys. Rev. Lett. 68, 3845 (1992).

[24] P. Adlarson et al. (WASA-at-COSY Collaboration), arXiv:1304.0671

[25] De-Chang Dai, K. Freese, and D. Stojkovic, JCAP 0906, 023 (2009).

[26] R. Essig, J. A. Jaros, W. Wester, P. H. Adrian, S. Andreas, T. Averett, O. Baker and B. Batell et al., arXiv:1311.0029

[27] R. Essig, J. Mardon, M. Papucci, T. Volansky and Y. M. Zhong, arXiv:1309.5084.

[28] J. S. Dworkin, P. T. Cox, E. C. Dukes, O. E. Overseth, R. Handler, R. Grobel, A. Jaske and B. Lundberg et al., Nucl. Instrum. Meth. A 247, 412 (1986).

[29] S. Andreas et al., arXiv:1312.3309.

[30] P. deNiverville, M. Pospelov and A. Ritz, Phys. Rev. D 84, 075020 (2011).

[31] H. Abramowicz et al. (CLIC Detector and Physics Study Collaboration), arXiv:1307.5288. 